FERMILAB-PUB-93/066-A

March 1993

\title{
Axion Miniclusters and Bose Stars
}

\author{
Edward W. Kolb ${ }^{(1),(2)}$ and Igor I. Tkachev ${ }^{(1),(3)}$ \\ (1) NASA/Fermilab Astrophysics Center \\ Fermi National Accelerator Laboratory, Batavia, IL 60510 \\ ${ }^{(2)}$ Department of Astronomy and Astrophysics, Enrico Fermi Institute \\ The University of Chicago, Chicago, IL 60637 \\ (3) Institute for Nuclear Research of the Academy of Sciences of Russia, Moscow \\ 117312, Russia
}

\begin{abstract}
Evolution of inhomogeneities in the axion field around the QCD epoch is studied numerically, including for the first time important nonlinear effects. It is found that perturbations on scales corresponding to causally disconnected regions at $T \sim 1 \mathrm{GeV}$ can lead to very dense axion clumps, with present density $\rho_{a} \gtrsim 10^{-8} \mathrm{~g} \mathrm{~cm}^{-3}$. This is high enough for the collisional $2 a \rightarrow 2 a$ process to lead to Bose-Einstein relaxation in the gravitationally bound clumps of axions, forming Bose stars.
\end{abstract}

PACS number(s): 98.80.Cq, 14.80.Gt, 05.30.Jp, 98.70.-f email: rocky@fnas01.fnal.gov; tkachev@fnas13.fnal.gov 
The invisible axion is one of the best motivated candidates for cosmic dark matter, despite being subject to strong cosmological and astrophysical constraints on its properties $\left(10^{10} \mathrm{GeV} \lesssim f_{a} \lesssim 10^{12} \mathrm{GeV}\right.$ for the axion decay constant; $10^{-5} \mathrm{eV} \lesssim m_{a} \lesssim$ $10^{-3} \mathrm{eV}$ for the axion mass) [1]. As dark matter, axions would play a role in the evolution of primordial density fluctuations and formation of large scale structure. In addition to its generic properties, axions also have unique features as dark matter. For instance, large amplitude density fluctuations produced on scales of the horizon at the QCD epoch [2] lead to tiny gravitationally bound "miniclusters" [3]. It was found that the density in miniclusters exceeds by ten orders of magnitude the local dark matter density in the Solar neighborhood [3]. This might have a number of astrophysical consequences, as well as implications for laboratory axion searches [4.

In previous studies, spatial gradients of the axion field in the equations of motion were neglected. This is a reasonable assumption for temperatures below the QCD scale where the evolution of coherent axion oscillations can be treated as pressureless, cold dust. However, we find that just at the crucial time when the inverse mass of the axion is approximately the size of the horizon, gradient terms become important, and a full field-theoretical approach is needed. Here we present the results of a numerical study of the evolution of the inhomogeneous axion field around the QCD epoch. Though we only consider spherically symmetric configurations, the importance of the combined effect of the field gradients and the non-liner attractive self interaction should also occur if we relax spherical symmetry. The resulting axion clumps are much denser than previously thought, reaching the critical conditions for Bose star formation [5].

The axion field $\theta(x)$ is created during the Peccei-Quinn symmetry breaking phase transition at $T \sim f_{a}$, uncorrelated on scales larger than the horizon at this time [6]. For $T \lesssim f_{a}$, the field becomes smooth on scales up to the horizon, $H^{-1}(T)$, where 
$H$ is the expansion rate. This continues until $T=T_{1} \approx 1 \mathrm{GeV}$ when the axion mass switches on, i.e., when $m_{a}\left(T_{1}\right) \approx 3 H\left(T_{1}\right)$. Coherent axion oscillations then transform fluctuations in the initial amplitude into fluctuations in the axion density.

Since the initial amplitude of coherent axion oscillations on the horizon scale $H^{-1}\left(T_{1}\right)$ is uncorrelated, one expects typical positive density fluctuations on this scale will satisfy $\rho_{a} \approx 2 \bar{\rho}_{a}$, where $\bar{\rho}_{a}$ is mean cosmological density of axions [3]. At the temperature of equal matter and radiation energy density, $T_{e}=5.5 \Omega_{a} h^{2} \mathrm{eV}$ [7], these fluctuations are already non-linear and will separate out as miniclusters with $\rho_{a} \approx 3(10 \mathrm{eV})^{4} \approx 10^{-14} \mathrm{~g} \mathrm{~cm}^{-3}$ [3]. The minicluster mass will be of the order of the dark-matter mass within the Hubble length at temperature $T_{1}, M_{\mathrm{mc}} \sim 10^{-9} M_{\odot}$. The radius of the cluster is $R_{\mathrm{mc}} \sim 10^{13} \mathrm{~cm}$, and the gravitational binding energy will result in an escape velocity $v_{e} / c \sim 10^{-8}$. Note that the mean phase-space density of axions in such a gravitational well is enormous: $n \sim \rho_{a} m_{a}^{-4} v_{e}^{-3} \sim 10^{48} f_{12}^{4}$, where $f_{12} \equiv f_{a} / 10^{12} \mathrm{GeV}$.

We will show below that due to non-linear effects, a substantial number of regions can have axion density at $T>T_{e}$ many times larger than $2 \bar{\rho}_{a}$.

Let us parametrize the energy density of a single fluctuation as $\rho_{a}\left(T_{e}<T<\right.$ $\left.T_{1}, \theta_{i}\right) \equiv 3 \Phi\left(\theta_{i}\right) T_{e} s / 4$, where $\theta_{i}$ is the misalignment angle at $T_{1}, s$ is the entropy density, and $\Phi\left(\theta_{i}\right)=1$ corresponds to the mean axion density. The energy density inside a given fluctuation is equal to the radiation energy density at $T=\Phi\left(\theta_{i}\right) T_{e}$. At that time the fluctuation becomes gravitationally non-linear and collapses. Consequently, at $T_{e}$

$$
\rho_{a}\left(\theta_{i}\right) \sim \Phi^{4}\left(\theta_{i}\right) \bar{\rho}_{a}\left(T_{e}\right)
$$

will be the minicluster density after it separates out as a bound object. Even a relatively small increase in $\Phi\left(\theta_{i}\right)$ is important because the density depends upon the 
fourth power of $\Phi\left(\theta_{i}\right)$.

Ref. [2] demonstrated that due to anharmonic effects for fluctuations with $\theta_{i}$ close to $\pi$, some correlated regions can have values of $\Phi\left(\theta_{i}\right)$ larger than just a factor of two. The reason is simple: the closer $\theta_{i}$ is to the top of the axion potential,

$$
V(\theta)=m_{a}^{2}(T) f_{a}^{2}(1-\cos \theta) \equiv \Lambda_{a}^{4}(T)(1-\cos \theta),
$$

the later axion oscillations commence. However this effect alone is not very significant. In the range $0.1 \lesssim \xi \lesssim 10^{-3}$ we can parametrize it as $\Phi\left(\theta_{i}\right) \approx 1.5\left(\theta_{i} / \pi\right)^{2} \xi^{-0.35}$, where $\xi \equiv\left(\pi-\theta_{i}\right) / \pi$, and $\Phi\left(\theta_{i}\right)$ is significantly larger than 2 only for field values very finely tuned to the top of the potential. Moreover, the axion field is not exactly coherent on the horizon scale, and small fluctuations might spoil this picture.

At temperatures $T \gg T_{1}$, the potential is negligible in the equations of motion compared to the gradient terms which force the field to be homogeneous on scales less than the horizon. At $T \ll T_{1}$, on the contrary, gradients can be neglected and one can treat the evolution of fluctuations as that of a pressureless gas. Clearly at $T \sim T_{1}$, both the gradient terms and the potential are important, and in order to find the energy density profile at freeze out one has to trace the inhomogeneous field evolution through the epoch $T \sim T_{1}$.

It is convenient to work in conformal coordinates with metric $d s^{2}=a^{2}(\eta)\left(d \eta^{2}-\right.$ $d \vec{x}^{2}$ ). During radiation dominance $a \propto \eta$ and $\eta \propto T^{-1}$. The dependence of the axion mass upon the temperature at $T>\Lambda_{\mathrm{QCD}}$ can be found in the dilute-instanton-gas approximation [8], and can be parametrized as a power law, $m_{a}^{2}(\eta)=m_{a}^{2}\left(\eta_{*}\right)\left(\eta / \eta_{*}\right)^{n}$, where $n=7.4 \pm 0.2$ [2]. Introducing the field $\psi=\eta \theta$, the equations of motion for a spherically symmetric axion fluctuation in an expanding Universe is of the form $\ddot{\psi}-\psi^{\prime \prime}-2 \psi^{\prime} / r+\bar{\eta}^{n+3} \sin (\psi / \eta)=0$, where $\bar{\eta}$ is the reduced conformal time parameter $\bar{\eta}=\eta / \eta_{*}$, and $m_{a}\left(\eta_{*}\right)=H\left(\eta_{*}\right)$. The radial coordinate $r$ is defined in the comoving 
FIG. 1. Energy density contrast in a fluctuation with initial radius $r_{0}=1.8$ and $\theta_{i}^{<}=2.75$ at several moments of time as a function of comoving radius $r$. The density contrast is normalized to the value of the homogeneous energy density at $\bar{\eta}=4$.

reference frame, with $r=1$ corresponding to $R_{\text {phys }}\left(\eta_{*}\right)=H^{-1}\left(\eta_{*}\right)$.

We integrated this equation numerically for a wide range of initial conditions. We evolved configurations which are at rest at $\bar{\eta}=0.1$. The initial distribution of the field can be parametrized by the initial radius of the fluctuation, $r_{0}$, the initial value of the field inside, $\theta_{i}^{<}$, the initial value of the field outside, $\theta_{i}^{>}$, and the width of transient region, $\Delta r$. The important common feature is that the final density distribution develops a sharp peak in the center. The larger the gradients of initial configuration, the higher the final peak, e.g., the peak grows with increase in $\left|\theta_{i}^{<}-\theta_{i}^{>}\right|$. 
FIG. 2. Energy density profiles at $\bar{\eta}=4$ for identical initial fluctuations evolved with different Lagrangians. Solid line: axion case; dashed line: $V(\theta) \propto \theta^{2} / 2$; dotted line: $V(\theta) \propto \theta^{2} / 2+\theta^{4} / 4$; dash-dotted line: axion potential with field gradients switched off.

The peak also grows with decreasing width of the transient region. We present here the results of runs with initial amplitude of the field outside the fluctuation equal to the r.m.s. value of the misalignment angle, i.e., $\theta_{i}^{>}=\pi / \sqrt{3}$, and width of transient layer $\Delta r \sim 0.6$.

Energy density profiles as a function of time are presented in Fig. 1 for a typical case. At $\bar{\eta}=1$ there are two waves, incoming and outgoing, both propagating with the velocity of light. At approximately $\bar{\eta}=2$ the incoming wave reaches the center and the outgoing wave reaches $r \approx 3.5$. At later times the wave front does not move 
significantly because the axion mass effectively switches on at $\bar{\eta} \approx 2$, and the edge of the fluctuation "freezes."

One reason for energy density growth at later times is the continuing increase of the axion mass. However the relative density contrast in the center with respect to the unperturbed homogeneous environment continues to increase up to the final time of integration, $\bar{\eta}=4$. This is entirely a non-linear effect. One can see this in the following way: The average pressure over a period of homogeneous axion oscillations in potential Eq. (2) is negative, and is equal to $\langle P\rangle \simeq-\Lambda_{a}^{4}(T) \theta_{0}^{4} / 64$, where $\theta_{0}$ is the amplitude of the oscillations [9]. In other words, the axion self-interaction is attractive. The larger the amplitude of oscillations inside the fluctuation, the more negative will be the pressure inside, and consequently, fluctuations with excess axions will contract in the comoving volume. In addition, matter with a smaller pressure suffers less redshift in the energy density. To see this effect we present in Fig. 2 the final density profiles correspondin g to identical initial field distributions evolved with different potentials: the axion potential of Eq. (2); the axion potential with gradients artificially switched off; a pure harmonic potential, $V(\theta) \propto \theta^{2} / 2$, where $\langle P\rangle=0$; and the potential $V(\theta) \propto \theta^{2} / 2+\theta^{4} / 4$, where $\langle P\rangle>0$. Note that for the harmonic potential, at $\bar{\eta}=4$ the maximal density excess is only about 3, i.e., ten times smaller than for the axion potential.

The dependence of the energy density contrast in the center upon the initial radius is shown on Fig. 3. In the whole range of values of $r_{0}$ plotted, the energy density takes its maximum value just in the center of the final configuration. Only if $r_{0}<1.55$ or $r_{0}>2.05$ does the final energy density profile have a maximum at some nonzero radius. In a sense, the initial radius of the fluctuation in the plotted range is more or less tuned in such a way that the arrival of the incoming wave at the center is synchronized with the switching on of the axion mass. However, there is nothing 
FIG. 3. Dependence of density contrast in the center of a fluctuation at $\bar{\eta}=4$ upon the initial radius of a fluctuation for several values of the initial misalignment angle inside the fluctuation.

unnatural in this "synchronization," since as larger and larger scales enter the horizon in an expanding Universe there will always be a scale for which the incident wave of a disappearing fluctuation reaches the center just at the moment of freeze out.

Quantitatively, the assumption of spherical symmetry is very important. However, in general any isolated contrast in the initial misalignment angle will decay via incoming and outgoing waves which will not possess spherical symmetry. The overall picture will be the same as in the spherical case, but the values of the maximal energy contrast in the final configuration at a given $\theta_{i}^{<}$will be smaller. Note in this respect, that the final density contrast rapidly grows with increase of $\theta_{i}^{<}$(see Fig. 3) due to the 
attractive self-interaction resulting in negative pressure. This has nothing to do with the symmetry of the fluctuation, and we may expect to find large density contrasts in regions where the field values happen to be close to $\pi$ initially [10].

The effect of the field gradients is important not only in the discussion of the formation of high density peaks, but also in the careful estimate of the mean density of axion matter. We found that the total excess mass of axions within a fluctuation, compared to the homogeneous background, does not vary much, and is equal approximately to half of the excess mass if gradients in the equations of motion would be neglected. This deficit might be attributed to the redshift at early times, $\bar{\eta} \lesssim 2$, when axions are still relativistic.

The energy density contrast plotted in Figs. 1 and 2 will coincide with the factor $\Phi\left(\theta_{i}\right)$ in Eq. (1]) if we assume that the mean cosmological density of axions corresponds to homogeneous oscillations with initial amplitude equal to the r.m.s. value of the misalignment angle. As we have noted already, the energy density in an axion clump after it separates out from the general expansion will be $\Phi^{4}\left(\theta_{i}\right)$ times larger than the energy density at $T_{e}$. So a density contrast of 30 will correspond to roughly a factor of $10^{6}$ in the energy density of the cluster at $T<T_{e}$.

All axion miniclusters could be, in principle, relevant to laboratory axion search experiments, since for a minicluster with $\Phi$ as small as 2 , the density is $10^{10}$ larger than the local galactic halo density. However, the probability of a direct encounter with a clump is small. The interesting question arises, could there be any astrophysical consequences of very dense axion clumps? Below we shall discuss the possibility of "Bose star" formation inside axion miniclusters.

The physical radius of an axion clump at $T_{e}$ is larger by many orders of magnitude than the de Broglie wavelength of an axion in the corresponding gravitational well. Consequently, gravitational collapse of the axion clump and subsequent virialization 
can be described in the usual terms of cold dark matter particles. In a few crossing times some equilibrium (presumably close to an isothermal) distribution of axions in phase space will be established. It is remarkable that in spite of the apparent smallness of axion quartic self-couplings, $\left|\lambda_{a}\right|=\left(f_{\pi} / f_{a}\right)^{4} \sim 10^{-53} f_{12}^{-4}$, the subsequent relaxation in an axion minicluster due to $2 a \rightarrow 2 a$ scattering can be significant as a consequence of the huge mean phase-space density of axions [5]. In the case of BoseEinstein statistics the inverse relaxation time is $(1+\bar{n})$ times the classical expression, or $\tau_{R}^{-1} \sim \bar{n} v_{e} \sigma \rho_{a} / m_{a}$, where $\sigma$ is the corresponding cross section. For part icles bounded in a gravitational well, it is convenient to rewrite this expression in the form [5

$$
\tau_{R} \sim m_{a}^{7} \lambda_{a}^{-2} \rho_{a}^{-2} v_{e}^{2}
$$

The shallower the gravitational well at a given density of axions, the larger the mean phase space density, and consequently the smaller the relaxation time due to the $v_{e}^{2}$ dependence in Eq. (3). Note also the dependence of the inverse relaxation time upon the square of the particle density.

The relaxation time (3) is smaller then the present age of the Universe if the energy density in the minicluster satisfies

$$
\rho_{10}>10^{6} v_{-8} \sqrt{f_{12}}
$$

where $\rho_{10} \equiv \rho /(10 \mathrm{eV})^{4}$ and $v_{-8} \equiv v_{e} / 10^{-8}$. If this occurs, then an even denser core in the center of the axion cloud should start to form. An analogous process is the so-called gravithermal instability caused by gravitational scattering. This was studied in detail for star clusters, where the "particles" obey classical Maxwell-Boltzmann statistics. Axions will obey Bose-Einstein statistics, with equilibrium phase-space density $n(p)=n_{\text {cond }}+\left[e^{\beta E}-1\right]^{-1}$, containing a sum of two contributions, a Bose condensate and a thermal distribution. The maximal energy density that non-condensed 
axions can saturate is $\rho_{\text {ther }} \sim m_{a}^{4} v_{e}^{3}$, which corresponds to $\bar{n}_{\text {ther }} \sim 1$. Consequently, given the initial condition $\bar{n} \gg 1$, one expects that eventually the number of particles in the condensate will be comparable to the total number of particles in the region where relaxation is efficient. Under the influence of self-g ravity, a Bose star [9,11] then forms [5]. One can consider a Bose star as coherent axion field in a gravitational well, generally with non-zero angular momentum in an excited energy state [9].

Comparing Eqs. (11) and (函), we conclude that the relaxation time is smaller than the present age of the Universe and conditions for Bose star formation can be reached in miniclusters with density contrast $\Phi\left(\theta_{i}\right) \gtrsim 30$ at the QCD epoch. For examples of such density contrasts, see Figs. 1 and 3.

Under appropriate conditions stimulated decays of axions to two photons in a dense axion Bose star are possible [9,12] (see also [13]), which can lead to the formation of unique radio sources - axionic masers. In view of results of present paper we conclude that the questions of axion Bose star formation, structure and possible astrophysical signatures deserves detailed study.

In conclusion, we have presented a numerical study of the evolution of inhomogeneties in the axion field around the QCD epoch, including for the first time important non-linear effects. We found that the non-linear effects can lead to a much larger core density of axions in miniclusters than previously estimated. The increase in the density may be sufficiently large that axion miniclusters might exceed the critical density necessary for them to relax to form Bose stars.

It is a pleasure to thank H. Feldman, J. Frieman, A. Kashlinsky, A. Klypin, D. Pogosyan, A. Stebbins, M. Turner, and R. Watkins for useful discussions. This work was supported in part by the DOE and NASA grant NAGW-2381 at Fermilab. 


\section{REFERENCES}

1. For recent reviews, see M. S. Turner, Phys. Rep. C197, 67 (1990); G. G. Raffelt, Phys. Rep. C198, 1 (1990).

2. M. S. Turner, Phys. Rev. D 33, 889 (1986).

3. C. J. Hogan and M. J. Rees, Phys. Lett. B205, 228 (1988).

4. P. Sikivie, Phys. Rev. Lett. 51, 1415 (1983).

5. I. I. Tkachev, Phys. Lett. B261, 289 (1991).

6. This does not necessarily require the reheating temperature after inflation to be higher than $f_{a}$, since inflation itself can produce strong fluctuations in the axion field as discussed in A. D. Linde and D. H. Lyth, Phys. Lett. B246, 353 (1990); D. H. Lyth and E. D. Stewart, Phys. Rev. D 46, 532 (1992).

7. E. W. Kolb and M. S. Turner, The Early Universe, (Addison-Wesley, Redwood City, Ca., 1990).

8. D. Gross, R. Pisarski, and L. Yaffe, Rev. Mod. Phys. 53, 43 (1981).

9. I. I. Tkachev, Sov. Astron. Lett. 12, 305 (1986).

10. We will discuss in a future paper the possibility that collapsing domain walls leave behind very dense clumps of non-relativistic axions.

11. R. Ruffini and S. Bonozzola, Phys. Rev. 187, 1767 (1969); J. D. Breit, S. Gupta, and A. Zaks, Phys. Lett. B140, 329 (1984).

12. I. I. Tkachev, Phys. Lett. B191, 41 (1987).

13. T. W. Kephart and T. J. Weiler, preprint VAND-TH-90-2, 1990 (unpublished). 